\title{
Análise de óleos-resinas de copaíba: contribuição para o seu controle de qualidade
}

\author{
Maique W. Biavatti ${ }^{1 *}$, Daniela Dossin ${ }^{1}$, Francisco C. Deschamps ${ }^{2}$, Maria da Paz Lima ${ }^{3}$ \\ ${ }^{1}$ Universidade do Vale do Itajaí, UNIVALI, Curso de Farmácia, Caixa Postal 360, \\ 88302-202, Itajaí, SC, Brasil, \\ ${ }^{2}$ Estação Experimental de Itajaí, EPAGRI, Rod. Antonio Heil, km 6, Caixa Postal 277, \\ 88301-970, Itajai, SC, Brasil, \\ ${ }^{3}$ Centro de Pesquisas em Produtos Naturais, Instituto Nacional de Pesquisas Amazônicas, CPPN-INPA, Caixa \\ Postal 476, 69083-000, Manaus, AM, Brasil
}

\begin{abstract}
RESUMO: A óleo-resina de copaíba pode ser extraída de forma sustentável do tronco de várias espécies de Copaifera. Ela contém principalmente ácidos resinosos e substâncias voláteis, tendo ampla utilização medicinal. A padronização cromatográfica do óleo é a garantia da identidade desta matéria-prima complexa. Neste trabalho analisaram-se por CG três amostras de óleos de copaíba obtidas de Colombo/PR e doze amostras adquiridas no Estado do Amazonas. O óleo de copaiba foi fracionado por cromatografia em sílica gel impregnada com $\mathrm{KOH}$ e metilado e as frações obtidas foram avaliadas por CG. Numa segunda etapa, os óleos brutos metilados por meio de diazometano ou transesterificados com $\mathrm{KOH} / \mathrm{MeOH}$ foram injetados diretamente em CG. Foram utilizados para comparação os ácidos diterpênicos hardwíckiico, copaiferólico e caurenóico além do sesquiterpeno 14-hidroxi- $\beta$-cariofileno (cariofilenol). A presença do ácido copaiferólico foi observada em $100 \%$ e cariofilenol em $80 \%$ das amostras. Os ácidos caurenóico e hardwíckiico ausentes nas amostras do Paraná, foram detectados em 8,3\% e 41,7\% respectivamente nas amostras do Amazonas. Os resultados obtidos indicam que podem ser analisados diretamente através de CG após metilação com diazometano, sem necessidade de fracionamento prévio. Adicionalmente, a determinação do índice de acidez para óleos de copaíba não demonstrou resultados inequívocos para as amostras analisadas
\end{abstract}

Unitermos: Copaifera, óleo de copaíba, cromatografia gasosa.

\begin{abstract}
Copaiba oil-resin analysis: contribution to quality control". Copaiba oil can be extracted from the trunk of some Copaifera species and contains mainly resinous acids and volatile substances, which have wide medicinal use. The chromatographic standardization of the oil characteristics is the guarantee of the identity of raw material. In this work, three copaiba oil samples from Colombo/PR, and twelve from Amazonas State were analyzed. The copaiba oil was fractionated in silica gel impregnated with $\mathrm{KOH}$, esterified and the fractions were evaluated by TLC and GC. In a second stage, the crude oil was esterified with diazomethane or transesterified with $\mathrm{KOH} / \mathrm{MeOH}$ and injected in GC. The diterpene acids used for comparison were hardwickiic, copaiferolic and caurenoic and the 14-hydroxy- $\beta$-caryophyllene (caryophyllenol). The presence of copaiferolic acid was observed in all analyzed samples and caryophyllenol in $80 \%$ of the samples. Hardwickiic and caurenoic acids were absent in the samples from Paraná State, and respectively detected in $8.3 \%$ and $41.7 \%$, of the samples from Amazonas. The obtained results indicate that the oil can be directly analyzed through GC after methylation with diazomethane, without previous fractionation. Also, an acidity index method proposed for copaiba oil analysis did not show to be reliable for the analyzed samples.
\end{abstract}

Keywords: Copaifera, gas chromatography, copaiba oil-resin.

\section{INTRODUÇÃO}

A origem do nome copaíba relaciona-se ao tupi "cupa-yba": a árvore de depósito, ou que tem jazida, em alusão clara ao óleo que guarda em seu interior. Popularmente conhecidas como copaibeiras ou paud'óleo, as espécies de copaíba são largamente distribuídas nas regiões amazônica e centro-oeste do Brasil, sendo o óleo de copaíba extraído de várias espécies de Copaifera (Leguminosae-Caesalpinoideae). No território brasileiro ocorrem mais de vinte espécies (Cascon; Gilbert, 2000) e entre as mais abundantes destacam-se $C$. officinalis L., C. guianensis Desf., C. reticulata Ducke, C. multijuga Hayne, $C$. confertiflora Bth., C. langsdorffii Desf., $C$. coriacea Mart., C. cearensis Huber ex Ducke (VeigaJúnior; Pinto, 2002). 
A coleta do óleo pode ser realizada de forma sustentável por incisão com trado a cerca de um metro de altura do tronco. O óleo de copaíba é um líquido transparente de viscosidade variável cuja coloração também pode variar, do amarelo ao marrom. Popularmente é utilizado como antiinflamatório (Braga et al., 1998; Cascon; Gilbert, 2000; Pinto et al., 2000), anti-séptico em feridas, eczemas, na psoríase e urticária (Cascon e Gilbert, 2000; Pinto et al., 2000), antiinflamatório das vias urinárias, em afecções pulmonares (tosses e bronquites, gripes e resfriados) (Paiva et al., 1998), cicatrizante de pequenas irritações do couro cabeludo, antiasmático, expectorante, na pneumonia, sinusite, disenteria, incontinência urinária, cistite e leucorréia (Veiga-Júnior; Pinto, 2002), como analgésico (Pinto et al., 2000), antiúlcera (Paiva et al., 1998), e como antitumoral (Ohsaki et al., 1994). O óleo de copaíba é utilizado também pelas indústrias de cosméticos e de vernizes como agente fixador (Veiga-Júnior; Pinto, 2002, Veiga-Júnior et al., 1997).

Até o momento, não foram encontrados estudos clínicos utilizando óleo de copaíba, porém alguns trabalhos sobre a atividade biológica em animais foram recentemente publicados, como atividade antitumoral (Lima et al., 2003), efeito protetor de colite aguda e do dano intestinal (Paiva et al., 2004a e 2004b) e antiinflamatório (Falcão et al 2005). Alguns poucos estudos químicos apontam sesqui e diterpenos como os principais componentes do óleo-resina das espécies de Copaifera (Arrhenius et al., 1983; Monti et al., 1996 e 1999, Braga et al., 1998; Cascon; Gilbert, 2000).

A carência de estudos químicos visando à caracterização do óleo de copaíba, aliada à variabilidade natural inerente a esta matéria-prima e à crescente demanda do mercado por medicamentos fitoterápicos, pode comprometer a autenticidade e a efetividade dos produtos contendo óleo de copaíba. Além disso, não há como garantir de maneira direta a identidade botânica da copaibeira, já que o óleo vem sendo obtido na maioria das vezes via extrativismo sustentável e, simultaneamente, de várias espécies de Copaifera. A falta de parâmetros efetivos para caracterizar e consequentemente realizar o controle de qualidade de óleo de copaíba é um dos principais entraves para o registro e exportação de produtos fitoterápicos contendo este insumo.

Alguns trabalhos analíticos recentes têm sido realizados buscando a padronização desta matériaprima, apesar de suas variações naturais, sendo que a cromatografia gasosa é a técnica mais empregada (Tappin et al., 2004, Cascon; Gilbert, 2000). Um método titulométrico alternativo para padronização do óleo de copaíba foi publicado recentemente (Vasconcelos; Godinho, 2002).

Neste trabalho obtiveram-se perfis cromatográficos através de cromatografia gasosa de várias óleos-resinas de copaíba autênticas $(C$. multijuga, coletadas em diferentes épocas) e comerciais provenientes do Estado do Amazonas, comparando com três lotes de óleo-resinas obtidas no Estado do Paraná. Os cromatogramas obtidos foram comparados com padrões dos diterpenos ácido hardwíckiico, ácido copaiferólico e ácido caurenóico, e com o sesquiterpeno cariofilenol, marcadores químicos deste insumo.

Foram também investigadas diferentes maneiras de preparação das amostras para análise em CG: fracionamento com sílica gel impregnada com

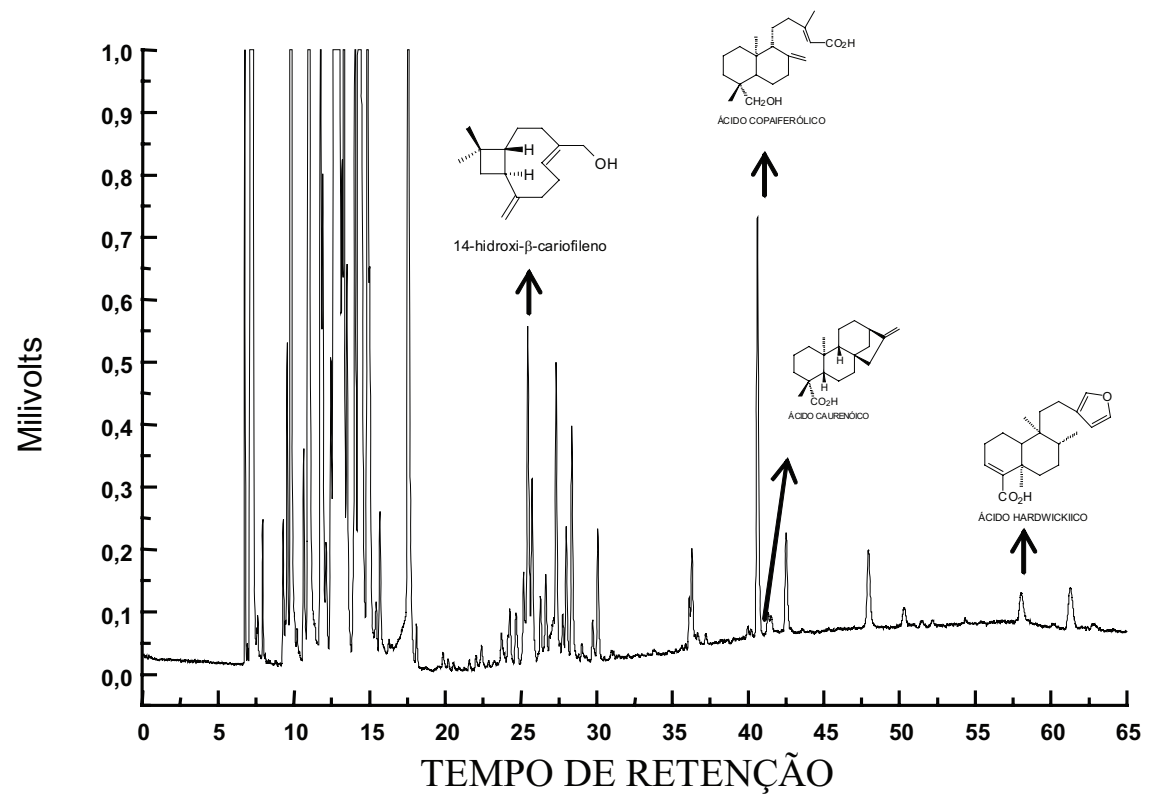

1. Oleo bruto metilado da amostra 14 (Paraná). Condições cromatográficas: materiais e (25.5 min); b. ácido copaiferólico (40.7 min); c. ácido caurenóico (41.3 6(2):abr/jun. 2006 


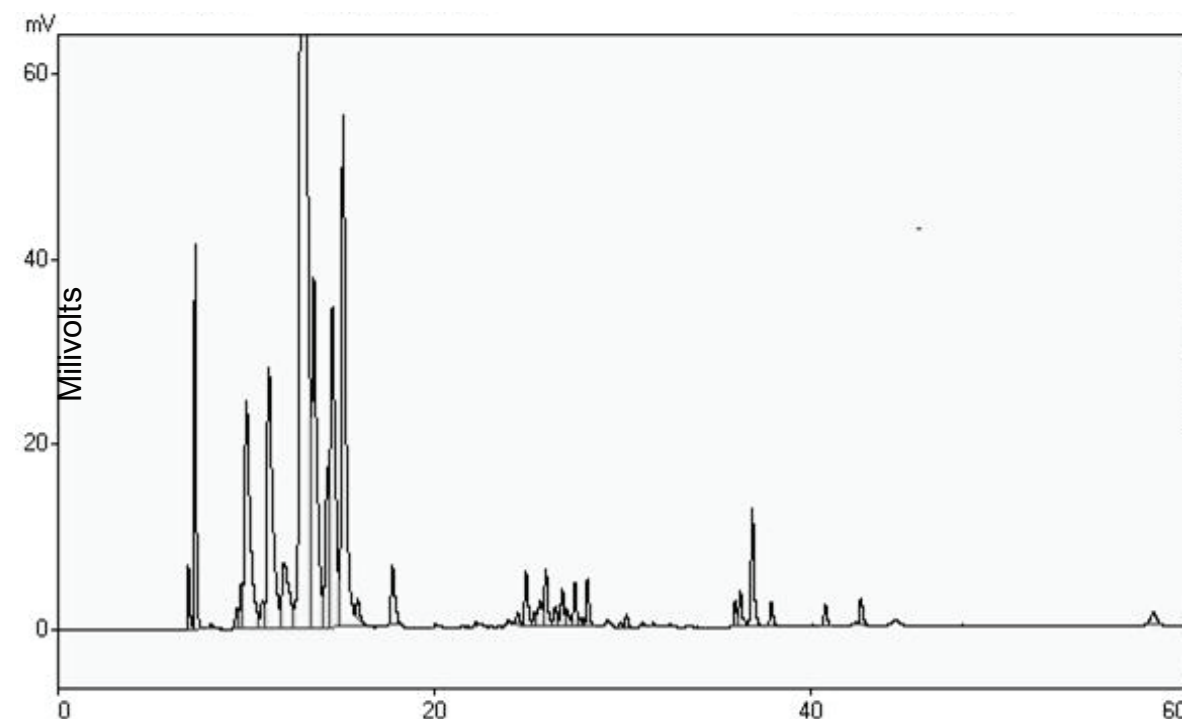

Figura 2. Óleo de copaíba bruto metilado da amostra 8 (Amazonas). Observam-se as três regiões características de eluição. Condições cromatográficas: materiais e métodos.

$\mathrm{KOH}$, metilação direta com diazometano e por meio de reação de transesterificação com $\mathrm{KOH}$ em $\mathrm{MeOH}$. Comparativamente, as amostras selecionadas de óleo de copaíba foram analisadas pelo método titulométrico proposto por Vasconcelos e Godinho (2002).

\section{MATERIAL E MÉTODOS}

\section{Obtenção das óleo-resinas de copaíba}

Amostras comerciais e autênticas (coletadas da espécie C. multijuga) de óleo-resinas de copaíba (OC) foram adquiridas no Estado do Amazonas (amostras 112) e obtidas através da FHSP (Colombo/PR) (amostras 13-15), de diversas procedências. As informações de cada amostra poderão ser disponibilizadas pelos autores. Todas as amostras foram mantidas refrigeradas durante a execução do trabalho. Os padrões de ácido hardwíckiico, ácido copaiferólico e cariofilenol, foram obtidos através de isolamento fitoquímico e identificados por métodos espectrométricos em comparação com dados da literatura.

\section{Cromatografia em coluna (CC)}

A amostra $13(5 \mathrm{~g})$ foi cromatografada na coluna impregnada com KOH utilizando-se como eluentes $400 \mathrm{ml}$ de $n$-hexano, diclorometano e metanol, respectivamente. Evaporaram-se os solventes e obtiveram-se as três frações: fração $n$-hexano (1,5 g), fração diclorometano $(2,0 \mathrm{~g}) \mathrm{e}$ fração metanol $(1,5 \mathrm{~g})$. A fração metanol foi acidificada com acido clorídrico $1 \%$ até $\mathrm{pH} 4,0$ e posteriormente extraída com diclorometano e evaporada $(1,2 \mathrm{~g})$ (Pinto et al., 2000).

\section{Preparação do diazometano para metilação das} amostras brutas

O diazometano foi preparado de acordo com o método descrito por Leonard et al. (1995). Para confirmar se o diazometano foi realmente produzido, uma alíquota do mesmo foi gotejado sobre um ácido carboxílico produzindo forte efervescência correspondente ao desprendimento de $\mathrm{N}_{2}$. As amostras de óleo bruto e a fração metanólica obtida acima foram metiladas utilizandose $1 \mathrm{ml}$ de amostra bruta diluída (ou padrão) em $1 \mathrm{ml}$ de diclorometano e $2 \mathrm{ml}$ de diazometano. As amostras filtradas $(0,45 \mu \mathrm{m})$ foram injetadas no cromatógrafo a gás um dia após a metilação.

\section{Metilação através de método alternativo}

Utilizou-se para fins comparativos o método descrito por Hartman e Lago (1973) para produzir ésteres metílicos das amostras brutas.

\section{Cromatografia gasosa (CG)}

As análises dos óleos por cromatografia gasosa de alta resolução (CGAR) foram realizadas em cromatógrafo Shimadzu, modelo GC-17A, equipado com detector de ionização de chama (DIC), e injetor automático AOC20I com volume de injeção de $1 \mu \mathrm{l}$ e razão de split de 100. Hélio foi utilizado como gás de arraste a uma vazão de $1,7 \mathrm{ml} \cdot \mathrm{min}^{-1}$. A programação da temperatura do forno foi de uma isoterma inicial de $5 \mathrm{~min}$ a $120{ }^{\circ} \mathrm{C}$ seguida de taxa de $4{ }^{\circ} \mathrm{C} . \mathrm{min}^{-1}$ até $240^{\circ} \mathrm{C}$ mantidos por 25 min. As temperaturas do injetor e do detector foram de $250^{\circ} \mathrm{C}$ e $260^{\circ} \mathrm{C}$, respectivamente. Em todas as análises foi 


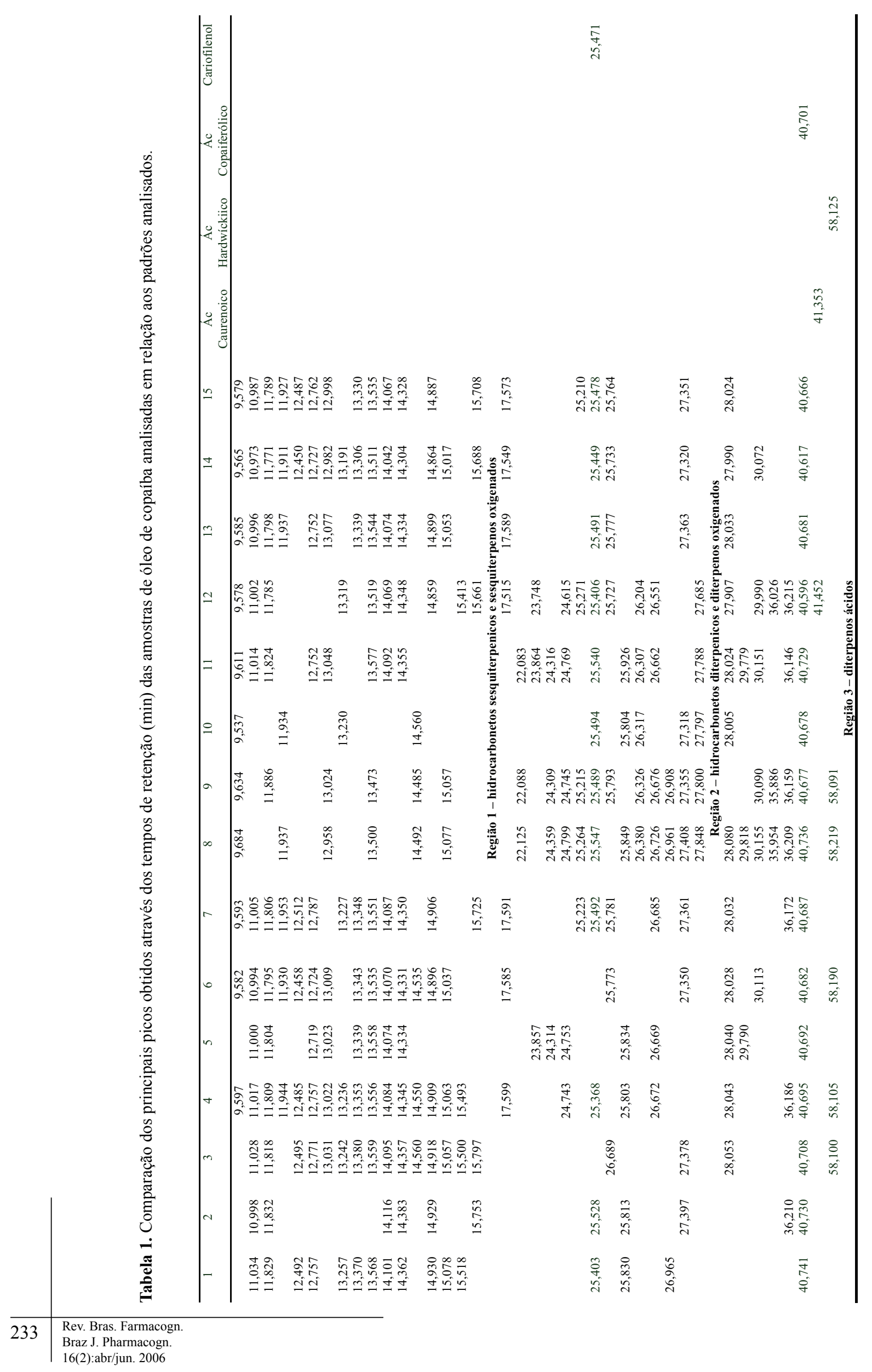


Tabela 2. Dados de índice de acidez (IA, valores médios, analises em duplicata) para amostras selecionadas segundo método descrito em Vasconcelos e Godinho (2002).

\begin{tabular}{|c|c|}
\hline Amostra & IA (mg KOH/g) \\
\hline Amostra 13 & 19,9 \\
\hline Amostra 14 & 20,5 \\
\hline Amostra 15 & 21,5 \\
\hline Amostra 8, Carauari/AM & 45,8 \\
\hline Amostra 9, Carauari/AM & 44,3 \\
\hline Amostra 10, Carauari/AM & 9,5 \\
\hline Amostra 11, Apuí/AM (C. multijuga) & 24,9 \\
\hline
\end{tabular}

utilizada coluna capilar de sílica SP2340 [100\% poli(biscianopropil-siloxano) - $60 \mathrm{~m} \times 0.25 \mathrm{~mm}$ id $\times 0.2 \mu \mathrm{m}]$ Todas as amostras foram filtradas através de membrana tipo Millex $(0,45 \mu \mathrm{m})$ antes da injeção.

Os picos referentes aos padrões foram identificados através de co-injeção e comparação dos tempos de retenção obtidos através do injetor automático.

\section{Índice de acidez}

Utilizou-se para fins comparativos o método descrito por Vasconcelos e Godinho (2002).

\section{RESULTADOS E DISCUSSÃO}

O fracionamento inicial por cromatografia flash em sílica impregnada com $\mathrm{KOH}$ visou facilitar a análise e a caracterização do óleo por cromatografia gasosa devido a sua complexidade, no entanto nos cromatogramas obtidos por CG para as frações hexano e diclorometano mostraram-se tão complexas quanto para os óleos brutos, sendo desnecessária portanto a separação previa do óleo bruto em coluna cromatográfica. A fração mais polar (metanolica metilada) forneceu as melhores condições para análise por apresentar menor sobreposição dos picos, sendo também a que possui componentes mais característicos de óleo de copaíba. O perfil cromatográfico por CG de uma amostra bruta de óleo de copaíba apresentou três regiões de eluição bem definidas, sendo a primeira mais congestionada e as duas últimas mais facilmente analisáveis, porém todas contendo abundantes picos. Seriam, pela ordem hidrocarbonetos sesquiterpênicos, sesquiterpenos oxigenados, hidrocarbonetos diterpênicos e diterpenos oxigenados (Pinto et al., 2000).

Pela analise das frações metanólicas dos óleos comerciais obtidos de Colombo (PR), observou-se a presença de poucos diterpenos, ao contrário do que se esperava conforme citado na literatura (Pinto et al., 2000). A análise direta por CG dos óleos brutos metilados apresentaram também um perfil carente de ácidos diterpênicos (Figura 1). Apenas duas amostras (amostras 8 e 9, que foram coletadas em épocas diferentes das demais) provenientes do Amazonas apresentaram o perfil próximo ao desejado por cromatografia gasosa, segundo a literatura (Figura 2). Esta observação confirma que a sazonalidade é um fator importante para a padronização da óleo-resina de copaíba. Sugere-se, portanto, que os fornecedores padronizem não apenas as características organolépticas do óleo coletado para formar seus lotes (por exemplo, a cor), mas também a época do ano em que o óleo foi coletado. Tradicionalmente, coletores relatam que a época reconhecida como ideal para a coleta da óleo-resina de copaíba é na lua cheia do mês de setembro (informação não publicada).

Comparando-se os diversos tempos de retenção obtidos por CG para todas as amostras analisadas, detectou-se a presença de ácido copaiferólico em todas as amostras, podendo este ser utilizado como um marcador quimiotaxonômico de óleos de copaíba por este ser característico e restrito ao gênero Copaifera. O cariofilenol foi observado nas amostras do Paraná e em cerca de $50 \%$ das amostras analisadas, porém os ácidos caurenóico e hardwíckiico não foram detectados, de acordo com os padrões utilizados (Tabela 1).

A tabela 1 mostra os principais picos obtidos para as amostras, em relação aos padrões analisados. A grande complexidade de insumos de origem natural, conduziu a OMS a considerar e aceitar técnicas cromatográficas fingerprint como uma maneira mais robusta de analisar a qualidade de produtos naturais, focalizando a comparação qualitativa e sistemática dos picos obtidos nas diferentes amostras. Desta maneira, a amostra é considerada como "substância ativa", facilitando a repetibilidade e a credibilidade de pesquisas clínicas e farmacológicas de insumos naturais (WHO, 1991). Neste sentido, três das amostras analisadas apresentaram menos de vinte substâncias detectadas, oito delas apresentaram entre 2026 picos, e quatro apresentaram mais de quarenta picos. Com isso observa-se a grande problemática envolvida na padronização do perfil ideal para o óleo de copaíba como insumo para a indústria farmacêutica.

Apesar da metodologia de transesterificação com $\mathrm{KOH} / \mathrm{MeOH}$ ser rápida e de baixo custo, nota-se que esta não é eficiente para os ácidos diterpênicos do óleo de copaíba como a metilação com diazometano. Esta conclusão deve-se a observação de que alguns compostos detectados por este segundo método não foram evidenciados no primeiro, como o ácido hardwickico e o cariofilenol. Pode-se sugerir com isso que a metilação por 
meio de diazometano é mais eficiente do ponto de vista reacional, ou então a etapa extrativa na transesterificação com $\mathrm{KOH} / \mathrm{MeOH}$ leva a perda de substâncias.

O método analítico clássico (índice de acidez) sugerido para óleo-resina de copaíba por Vasconcelos e Godinho (2002) foi aplicado em algumas amostras selecionadas, por necessitar um maior volume para as análises. Este é um método titulométrico tradicional e de baixo custo para investigação de adulterações grosseiras do óleo de copaíba. Foram selecionadas as amostras procedentes do Paraná e procedentes de Carauari (AM) (com grau de complexidade maior, segundo análise por CG), porém, não se obteve os índices de acidez esperados segundo a proposição do autor (maior que $80 \mathrm{mg} \mathrm{KOH} / \mathrm{g}$ ) para nenhuma das amostras testadas, inclusive a autêntica (Tabela 2). Apesar de ser um método acessível do ponto de vista econômico, não conduz a resultados inequívocos, contradizendo a conclusão dos autores, que o julgaram eficiente. Devido aos resultados encontrados neste trabalho, acredita-se que este método deve ser avaliado contemplando um numero maior de amostras, antes de ser sugerido como método para controle de qualidade de óleos de copaíba.

Este trabalho utilizou pela primeira vez como marcadores de amostras de óleo de copaíba o ácido copaiferólico e o cariofilenol, sendo o primeiro encontrado em todas as amostras analisadas por cromatografia gasosa, e testou comparativamente método para determinação titulométrica do índice de acidez para detecção de substâncias adulterantes, o qual não se mostrou eficaz.

\section{AGRADECIMENTOS}

Os autores agradecem o apoio da Fundação Herbarium de Saúde e Pesquisa (FHSP), INPA (AM), EPAGRI (SC), CNPq - EDITAL FITOTERÁPICOS, e os pesquisadores Prof. Dr. Franco Delle Monache, Centro Nazionale di Ricerca (CNR), Roma, Itália e Prof. Dr. Valdir Cechinel Filho, UNIVALI que gentilmente cederam padrões para as análises.

\section{REFERÊNCIAS}

Arrhenius SP, Foster CE, Edmonds CG, Langenheim JH 1983. Sesquiterpenes in leaf pocket resins of Copaifera species. Phytochemistry 22: 471-472.

Braga WF, Rezende CM, Antunes AC, Pinto AC 1998. Terpenoids from Copaiba cearensis. Phytochemistry 49: 263-264.

Cascon V, Gilbert B 2000. Characterization of the chemical composition of oleoresins of Copaifera guianensis Desf., Copaifera duckei Dwyer and Copaifera multijuga Hayne. Phytochemistry 55: 773-778.

Falcão HS, Lima IO, Santos VL, Dantas HF, Diniz MFFM, Barbosa-Filho JM, Batista LM 2005. Review of the plants with anti-inflammatory activity studied in Brazil. Rev Bras Farmacogn 15: 381-391.

Hartman L, Lago RCA 1973. Rapid preparation of fatty acid methyl esters from lipids. Lab Pract 22: 475-476.

Leonard J, Lygo B, Procter G 1995. Advanced Practical Organic Chemistry. $2^{\mathrm{a}}$ ed. Oxford: Chapman \& Hall, $298 \mathrm{p}$.

Lima SR, Veiga-Júnior VF, Christo HB, Pinto AC, Fernandes PD 2003. In vivo and in vitro studies on the anticancer activity of Copaifera multijuga Hayne and its fractions. Phytother Res 17: 1048-1053.

Monti H, Tiliacos N, Faure R 1996. Two diterpenoids from copaiba oil. Phytochemistry 42: 1653-1656.

Monti H, Tiliacos N, Faure R 1999. Copaiba oil: isolation and characterization of a new diterpenoid with the dinorlabdane skeleton. Phytochemistry 51: 10131016.

Ohsaki A, Yan LT, Ito S, Edatsugi H, Iwata D, Komoda Y 1994. The isolation and in vivo potent antitumour activity of clerodane diterpenoid from the oleoresin of the Brazilian medicinal plant, Copaifera langsdorffii Desfon. Bioorg Med Chem Lett 4: 2889-2892.

Paiva LA, Rao VS, Gramosa NV, Silveira ER 1998. Gastroprotective effect of Copaifera langsdorffii oleoresin on experimental gastric ulcer models in rats. $J$ Ethnopharmacol 62: 73-78.

Paiva LA, Gurgel LA, De Sousa ET, Silveira ER, Silva RM, Santos FA, Rao VS 2004a. Protective effect of Copaifera langsdorffii oleo-resin against acetic acidinduced colitis in rats. $J$ Ethnopharmacol 93: 51-56.

Paiva LA, Gurgel LA, Campos AR, Silveira ER, Rao VS 2004b. Attenuation of ischemia/reperfusion-induced intestinal injury by oleo-resin from Copaifera langsdorffii in rats. Life Sci 75:1979-1987.

Pinto AC, Braga WF, Rezende CM, Garrido FMS, Veiga Júnior VF, Bergyer L, Patitucci ML, Antunes AC 2000. Separation of acid diterpenes of Copaifera cearensis Huber ex Duke by flash chromatography using potassium hidroxide impregnated silica gel. $J$ Braz Chem Soc 11: 355-360.

Tappin MRR, Pereira JFG, Lima LA, Siani AC, Mazzei JL, Ramos MFS 2004. Análise química quantitativa para a padronização do óleo de copaíba por cromatografia em fase gasosa de alta resolução. Quim Nova 27: 236240.

Vasconcelos AFF, Godinho OES 2002. Uso de métodos analíticos convencionais no estudo da autenticidade do óleo de copaíba. Quim Nova 25: 1057-1060.

Veiga-Júnior VF, Patitucci ML, Pinto AC 1997. Controle de autenticidade de óleo de copaíba comerciais por cromatografia gasosa de alta resolução. Quim Nova 20: 612-615.

Veiga-Júnior VF, Pinto AC 2002. O gênero Copaifera L. Quim Nova 25: 273-286.

World Health Organization, Guidelines for the Assessment of Herbal Medicines, Munich, 28.6.1991, WHO, Geneva, 1991. 